\title{
Successful osimertinib rechallenge following drug-induced pneumonitis after previous anti-PDL1 exposure
}

\author{
Guilherme Harada ${ }^{1}$, Fernando Costa Santini ${ }^{1}$, Felipe Sales Nogueira Amorim Canedo ${ }^{1}$, Leandro Jonata de Carvalho Oliveira ${ }^{1}$, \\ Henrique Bortot Zuppani ${ }^{2}$ and Gilberto De Castro Jr ${ }^{1}$ \\ ${ }^{1}$ Hospital Sírio Libanês, São Paulo 01525-001, Brazil \\ ${ }^{2}$ Instituto do Câncer do Estado de São Paulo, São Paulo 01525-001, Brazil
}

\section{Abstract}

Osimertinib is a first-line treatment option for patients with metastatic non-small cell lung cancer (NSCLC) harbouring EGFR mutations. Pneumonitis is a severe adverse event $(\mathrm{AE})$ related to osimertinib treatment which appears to be more frequent when associated with concurrent or previous anti-PD(L)1 exposure. Data regarding the efficacy and safety of osimertinib rechallenge, especially in the setting of central nervous system (CNS) metastases, are scarce. We herein describe a case of a 53-year-old patient with metastatic EGFR-mutated NSCLC, who developed pneumonitis after osimertinib treatment and was successfully rechallenged with $40 \mathrm{mg}$ daily osimertinib, with CNS response. This dose reduction strategy may be an option for selected patients with brain metastases after tyrosine kinase inhibitors-induced AEs.

Keywords: lung cancer, osimertinib, immunotherapy, pneumonitis, rechallenge, brain metastases

\section{Introduction}

Brain metastases (BMs) occur initially in $20 \%$ of patients with non-small cell lung cancer (NSCLC) and 30\%-50\% of patients will be diagnosed with BMs during the course of their disease [1, 2]. In patients with EGFR-mutated tumours, the incidence of BMs at the time of diagnosis is higher than in unselected patients (25\%), suggesting that EGFR mutations might be associated with a metastatic tropism to the brain [3].

Among patients with NSCLC with EGFR mutations, tyrosine kinase inhibitors (TKIs) seem effective in controlling the intracranial disease. Preclinical and clinical evidence indicate that EGFR-TKIs of the third generation are more effective in treating BMs than their first- and second-generation counterparts, showing a promising blood-brain barrier (BBB) penetration and the potential to overcome EGFR-TKI resistance [4].

Lung abnormalities, from transient asymptomatic pulmonary opacities to full-blown interstitial lung disease, have been described as adverse events (AEs) associated with osimertinib, a third-generation EGFR-TKI. Severe AEs have been reported when EGFR-TKIs (especially osimertinib) follow immunotherapy [5]. Potential interactions of these therapies can cause unpredicted overlapping AEs.
Correspondence to: Guilherme Harada Email: guiarada@hotmail.com

ecancer 2019, 13:970

https://doi.org/10.3332/ecancer.2019.970

Published: $21 / 10 / 2019$

Received: 02/07/2019

Publication costs for this article were supported by ecancer (UK Charity number 1176307).

Copyright: $(\subset)$ the authors; licensee ecancermedicalscience. This is an Open Access article distributed under the terms of the Creative Commons Attribution License (http:// creativecommons.org/licenses/by/3.0), which permits unrestricted use, distribution, and reproduction in any medium, provided the original work is properly cited. 
We hereby describe a case of successful half-dose osimertinib rechallenge with central nervous system (CNS) response in a patient with EGFR-mutated NSCLC, previously treated with an anti-PD-L1 antibody, which developed severe pneumonitis after initial osimertinib exposure.

\section{Case report}

Here, we report a 53-year-old male, never-smoked patient, who had undergone left lower lobectomy and mediastinal sampling for lung adenocarcinoma, pathologic stage IIIC (T3N3M0), harbouring an EGFR exon 19 deletion (p.Leu747_Ala750delinsPro; NM_00528), detected by pyrosequencing (PyroMark Q24, Qiagen, Tokyo, Japan). Post-surgical 18-fluorodeoxyglucose positron emission tomography/computerized tomography revealed positive upper and lower contralateral (right) paratracheal nodes. Definitive concurrent chemoradiation (54 Gy delivered concurrently with cisplatin $50 \mathrm{mg} / \mathrm{m}^{2}$ on days $1,8,29$ and 36 , plus etoposide $50 \mathrm{mg} / \mathrm{m}^{2}$ daily on days $1-5$ and 29-33) was administered up to February 2018, followed by durvalumab $10 \mathrm{mg} / \mathrm{kg}$, starting in April 2018. After eleven 14-day cycles, he complained of a new-onset headache. Brain MRI revealed widespread supra and infratentorial brain parenchymal metastasis, and he underwent surgical resection of two bilateral frontal metastases (left $4.1 \mathrm{~cm}$ and right $2.8 \mathrm{~cm}$ ), which confirmed EGFR-mutant lung adenocarcinoma, harbouring the same EGFR exon 19 deletion (p.Leu747_Ala750delinsPro; NM_00528). Twenty-two days after durvalumab interruption, osimertinib 80 mg once daily was initiated. On the 53rd day of osimertinib treatment, the patient was admitted due to intense dyspnoea on exertion and cough. Chest CT scan revealed patchy ground-glass opacities (Figure 1). As interstitial lung disease induced by osimertinib was considered, EGFR-TKI was suspended and prednisone $1 \mathrm{mg} / \mathrm{kg} / \mathrm{day}$ was started, along with piperacillin-tazobactam. Three weeks later, a new CT scan showed significant improvement and all symptoms subsided. Patient was rechallenged with osimertinib $40 \mathrm{mg}, 42$ days after its suspension, and prednisone was rapidly tapered down. As of today, 2 months after this rechallenge, new brain MRI showed tumour response, including shrinkage in the right occipitotemporal lesion $(0.8 \mathrm{~cm} \times 0.5 \mathrm{~cm}$; previously $1.8 \mathrm{~cm} \times 1.4 \mathrm{~cm}$ ) (Figure 2) and the left cerebellar lesion $(0.5 \mathrm{~cm}$; previously $1.1 \mathrm{~cm} \times 0.8 \mathrm{~cm})$. No signs of pneumonitis recurrence were noticed.

\section{Discussion}

Osimertinib is a treatment option in the first-line setting for patients with metastatic EGFR-mutated NSCLC [6]. It achieves greater intracranial concentrations than first- and second-generation EGFR-TKIs [7] and exhibited a longer progression-free survival (PFS) (15.2 months versus 9.6 months; hazard ratio $0.47,95 \%$ confidence interval $(\mathrm{Cl}) 0.30-0.74)$ in a subset analysis of the FLAURA trial, which included 116 treatment-naïve patients with EGFR-mutated advanced NSCLC and CNS metastases [8]. In the FLAURA trial, osimertinib showed a lower rate of CNS progression (6\% versus $15 \%$ ) and an increased intracranial response rate (91\% versus $68 \%$ ) than first-line treatment with gefitinib or erlotinib [6].
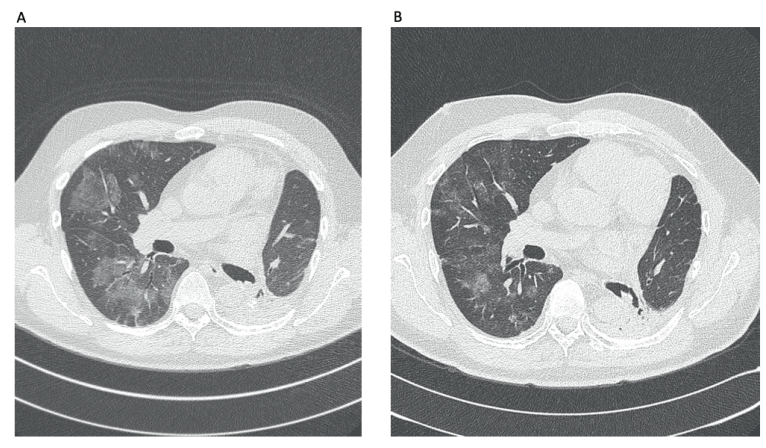

Figure 1. Chest CT findings before and after corticotherapy. (A): Patchy ground-glass opacity, mainly in the right lung. (B): Significant improvement after 3 weeks with prednisone. 

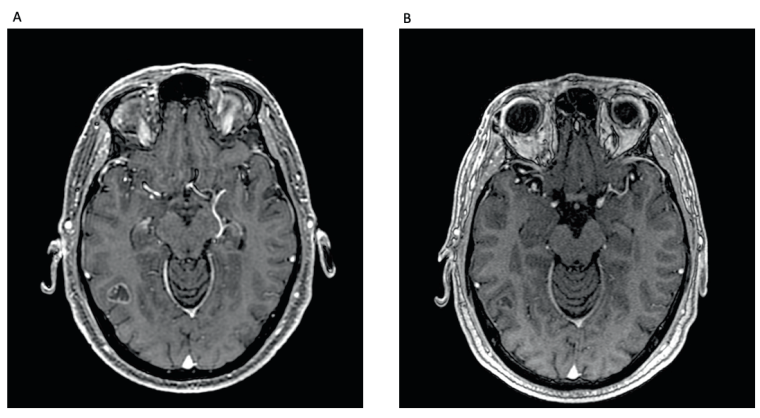

Figure 2. (A): Brain MRI demonstrating right occipitotemporal lesion measuring $1.8 \mathrm{~cm} \times 1.4 \mathrm{~cm}$. (B): Two months after rechallenge of osimertinib and reduction of the lesion, measuring $0.8 \mathrm{~cm} \times 0.5 \mathrm{~cm}$.

In order to optimise the CNS control in EGFR-mutated patients, the combination of targeted therapies and radiotherapy has been studied. Radiotherapy is known to disrupt BBB and potentially increases the effect of EGFR-TKIs in CNS, even if such inhibitors already penetrate the BBB [2]. A retrospective multi-institutional analysis suggested that stereotactic radiosurgery (SRS) followed by first-generation EGFR-TKI resulted in longer overall survival (OS) when compared with EGFR-TKI followed by SRS or whole-brain radiotherapy at progression [9]. Regarding the association of osimertinib and radiotherapy, Xie et al did not demonstrate superiority in time to treatment failure, PFS and OS of association of radiotherapy and osimertinib compared to osimertinib alone in a retrospective analysis [10]. Osimertinib has not been evaluated with SRS in prospective trials yet, and additional studies are needed to address these questions. A clinical trial is open to evaluate osimertinib with or without SRS for EGFR-mutated NSCLC with BM (NCT03497767).

The increase of osimertinib toxicity in patients who used anti-PD(L)1 was recently described, and interstitial lung disease is one of the main AEs [5]. The concomitant or sequential use of osimertinib and durvalumab was also associated with a high risk of pneumonitis [11, 12], confirming that this association is related to a higher risk of immunotoxicity. It is important to highlight that this overlapping toxicity seems to be osimertinib-related, rather than class-specific, since the association of other EGFR-TKIs and anti-PD(L)1 did not present the same toxicity rates [13].

Osimertinib rechallenge is an important point to be considered. A successful full-dose osimertinib rechallenge has been reported in a patient with BMs following salvage cytotoxic chemotherapy, showing potential intracranial therapeutic effect with re-exposure to the drug [14]. Metro et al described a case of an EGFR T790M-positive lung cancer patient, who was pretreated with the sequence erlotinib-osimertinib and experienced a dramatic response to osimertinib rechallenge after intervening chemotherapy [15]. In a retrospective analysis, 17 patients were rechallenged with osimertinib after acquiring resistance. The objective response was $33 \%$ and disease control rates were $73 \%$. The median PFS was 4.1 months ( $95 \% \mathrm{Cl}$ : 1.9-6.7). The toxicity was low, being that most patients had grade 2 adverse events (AEs) or lower, without interruption of treatment due to AEs [16].

Previous experiences indicate that re-administration of $40 \mathrm{mg}$ osimertinib may be a safe and effective strategy in patients who developed osimertinib-induced pneumonitis with standard-dose use [17]. Clinicians must be aware of osimertinib potential toxicities and of viable strategies to manage them so as to guarantee maximum benefit to metastatic EGFR-mutated NSCLC patients. The present case indicates that it may be feasible to treat patients with BMs with osimertinib 40 mg daily if dose reduction is needed after a severe drug-related AE.

\section{Conclusion}

To the authors' knowledge, this is the first report of activity with CNS response and safety after rechallenge with osimertinib 40 mg. The safest time interval between interrupting immunotherapy and starting osimertinib is still an unanswered question. Once patients with BMs have the urgency to start a CNS active therapy and considering the efficacy of osimertinib in EGFR-mutant NSCLC, osimertinib rechallenge can be considered in selected cases. 


\section{Conflicts of interest}

Dr de Castro Junior reports personal fees and other from AstraZeneca, personal fees and other from Roche, personal fees and other from Boehringer-Ingelheim, outside the submitted work.

The other authors declare that they have no conflicts of interest.

\section{Funding statement}

No funding was received.

\section{References}

1. Barnholtz-Sloan JS, Sloan AE, and Davis FG, et al (2004) Incidence proportions of brain metastases in patients diagnosed (1973 to 2001) in the Metropolitan Detroit Cancer Surveillance System J Clin Oncol 22 2865-2872 https://doi.org/10.1200/JCO.2004.12.149 PMID: 15254054

2. Preusser M, Winkler F, and Valiente M, et al (2018) Recent advances in the biology and treatment of brain metastases of non-small cell lung cancer: summary of a multidisciplinary roundtable discussion ESMO Open 3 e000262 https://doi.org/10.1136/esmoopen-2017-000262 PMID: 29387475 PMCID: 5786916

3. Bhatt VR, Kedia S, and Kessinger A, et al (2013) Brain metastasis in patients with non-small-cell lung cancer and epidermal growth factor receptor mutations J Clin Oncol 31 3162-3164 https://doi.org/10.1200/JCO.2013.49.8915 PMID: 23897953

4. Kim DW, Yang JC-H, and Chen K, et al (2015) AZD3759, an EGFR inhibitor with blood brain barrier (BBB) penetration for the treatment of non-small cell lung cancer (NSCLC) with brain metastasis (BM): preclinical evidence and clinical cases J Clin Oncol 33(15_suppl) 8016 https://doi.org/10.1200/jco.2015.33.15_suppl.8016

5. Schoenfeld AJ, Arbour KC, and Rizvi H, et al (2019) Severe immune related adverse events are common with sequential PD-(L)1 blockade and osimertinib Ann Oncol 30(5) 839-844 https://doi.org/10.1093/annonc/mdz077 PMID: 30847464

6. Soria JC, Ohe Y, and Vansteenkiste J, et al (2018) Osimertinib in untreated EGFR-mutated advanced non-small-cell lung cancer N Engl J Med 378(2) 113 https://doi.org/10.1056/NEJMoa1713137

7. Ballard P, Yates JWT, and Yang Z, et al (2016) Preclinical comparison of osimertinib with other EGFR-TKIs in EGFR-mutant NSCLC brain metastases models, and early evidence of clinical brain metastases activity Clin Cancer Res 20(22) 5130-5140 https://doi. org/10.1158/1078-0432.CCR-16-0399

8. Reungwetwattana T, Nakagawa K, and Cho BC, et al (2018) CNS response to osimertinib versus standard epidermal growth factor receptor tyrosine kinase inhibitors in patients with untreated EGFR-mutated advanced non-small-cell lung cancer J Clin Oncol 36(33) 3290-3297 https://doi.org/10.1200/JCO.2018.78.3118

9. Magnuson WJ, Lester-Coll NH, and Wu AJ, et al (2017) Management of brain metastases in tyrosine kinase inhibitor-naïve epidermal growth factor receptor-mutant non-small-cell lung cancer: a retrospective multi-institutional analysis J Clin Oncol $351070-1077$ https://doi.org/10.1200/JCO.2016.69.7144 PMID: 28113019

10. Xie L, Nagpal S, and Wakelee HA, et al (2019) Osimertinib for EGFR-mutant lung cancer with brain metastases: results from a single-center retrospective study Oncologist 24(6) 836-843 https://doi.org/10.1634/theoncologist.2018-0264 
11. Yang JCH, Shepherd FA, and Kim DW, et al (2019) Osimertinib plus durvalumab versus osimertinib monotherapy in EGFR T790Mpositive NSCLC following previous EGFR TKI therapy: CAURAL brief report J Thorac Oncol 14(5) 933-939 https://doi.org/10.1016/ j.jtho.2019.02.001

12. Ahn MJ, Yang J, and Yu H, et al (2016) 1360: osimertinib combined with durvalumab in EGFR-mutant non-small cell lung cancer: results from the TATTON phase Ib trial J Thorac Oncol 11(4) S115 https://doi.org/10.1016/S1556-0864(16)30246-5

13. Gettinger S, Hellmann MD, and Chow LQM, et al (2018) Nivolumab plus erlotinib in patients with EGFR-mutant advanced NSCLC J Thorac Oncol 13(9) 1363-1372 https://doi.org/10.1016/j.jtho.2018.05.015 PMID: 29802888

14. Sekine A, Satoh H, and Ikeda S, et al (2019) Rapid effect of osimertinib re-challenge on brain metastases developing during salvage cytotoxic chemotherapy after osimertinib treatment failure: a case report Mol Clin Oncol 10(4) 451-453 PMID: 30931116 PMCID: 6425508

15. Metro G, Baglivo S, and Siggillino A, et al (2018) Successful response to osimertinib rechallenge after intervening chemotherapy in an EGFR T790M-positive lung cancer patient Clin Drug Investig 38(10) 983-987 https://doi.org/10.1007/s40261-018-0691-8 PMID: 30151614

16. Ichira E, Hotta K, and Nimomiya K, et al (2019) Re-administration of osimertinib in osimertinib-acquired resistant non-small-cell lung cancer Lung Cancer 132 54-58 https://doi.org/10.1016/j.lungcan.2019.02.021

17. Satoh S, Shiroyama T, and Tamiya M, et al (2018) Successful osimertinib rechallenge after osimertinib-induced pneumonitis in a patient with lung adenocarcinoma Respir Med Case Rep 23 68-70 PMID: 29487786 PMCID: 5805846 